\title{
Antenna array design by a contraction adaptive particle swarm optimization algorithm
}

\author{
Xin Zhang ${ }^{1,2}$, Dunqiang Lu ${ }^{1,2}$, Xiu Zhang ${ }^{1,2^{*}}$ (D) and Yue Wang ${ }^{1,2}$
}

\begin{abstract}
Massive multiple input multiple output (MIMO) has drawn intensive attention of researchers for solving huge data transmissions in the fifth generation wireless communication. Antennas aligning in array bring in advantages for interference reduction of incoming wave signals and interference offset among antenna elements. The design of antenna array is studied in this paper. The design is turned into a maximization problem. A contraction adaptive particle swarm optimization (CAPSO) method is proposed to solve the problem. Different from previous methods, CAPSO is based on a contraction factor which limits the variation neighborhood during the solution searching process. The adaptive technique can tune the searching range of CAPSO. Simulations are reported comparing the CAPSO method with other methods. Convergence rate is analyzed through two toy functions. CAPSO shows a fast convergence property. Then it is used to solve antenna array design. The CAPSO method shows good performance for different numbers of array elements.
\end{abstract}

Keywords: Antenna array, MIMO, Particle swarm optimization, Numerical optimization

\section{Introduction}

Massive multiple input multiple output (MIMO) is a hot topic for providing good quality of service (QoS) in the fifth generation (5G) wireless mobile communication [1]. Not only data transmission efficiency but also transmission reliability and privacy security have to be considered to assure good QoS [2,3]. Moreover, new communication technology is also welcome such as cooperative communication, heterogeneous network, and compressive sensor network [4-6]. Among different network structures, resource allocation, network efficiency, throughput, and other aspects need to be studied as soon as possible [7, 8].

Antenna is essential in physical layer communication to assure data transmission in $5 \mathrm{G}$ wireless communication [9]. Antenna array is even more useful due to high gain and reliability of arranging many elements [10]. Optimal antenna design methods can be classified to deterministic methods and heuristic methods. Deterministic methods

\footnotetext{
*Correspondence: zhang210@126.com

${ }^{1}$ Tianjin Key Laboratory of Wireless Mobile Communications and Power Transmission, Tianjin Normal University, Tianjin, China

${ }^{2}$ College of Electronic and Communication Engineering, Tianjin Normal University, Tianjin, China
}

often converge very quickly but requiring prior knowledge of geometric structure of antennas [11-13]. To solve difficult design models, relaxation techniques are generally utilized to reduce the complexity of models [14-16]. Parallel computing, matrix decomposition, and other methods are also utilized to speed up the convergence rate for deterministic methods [17-21].

Heuristic algorithms for antenna design include genetic algorithm (GA) [22], particle swarm optimization (PSO) [23], differential evolution [24, 25], and neighborhood field optimization [26]. Recently, improved heuristic algorithms have been used to design wireless communication network designs, power allocation, and antennas [27, 28].

PSO has been studied and applied to many different problems in the real world [29]. Since its creation, standard PSO has been improved from many aspects including parameter control, search equation, and neighborhood network structure. This paper attempts to tune algorithmic parameters based on contraction and accelerating factors.

The above is discussed for the purpose of the paper. There are two contributions in the paper. The first is 
the improved parameter adaptation based on contraction, which improves the form of the iterative formula for the PSO algorithm. Another one is the convergence rate analysis and application to antenna design with different numbers of antenna elements.

Section 3 briefly introduces the antenna design problem and related works. Section 4 introduces standard PSO and the proposed contraction adaptive particle swarm optimization (CAPSO) algorithms. Section 6 reports simulation results compared with other methods, and the paper is concluded in Section 7.

\section{Method and experiment}

The study of the paper is to effectively solve antenna array designs. The study is accomplished by two parts. First, antenna array designs are expressed as an optimization problem. The problem model contains the main factors in antenna array designs. Second, a contraction adaptive particle swarm optimization algorithm is used to solve the problem model. The efficiency of the algorithm should be analyzed to assure the problem model being effectively solved. Comparisons are made by taking three algorithms which are of the same type as the proposed CAPSO algorithm. Comparison results show that the CAPSO algorithm is more efficient than other algorithms. Then, it is used to solve antenna array designs. The experiment is based on numerical simulations. To verify the effectiveness of the proposed method, designs are modeled as easy to use and scalable type. The analysis shows that the proposed method is useful and effective to solve antenna array designs.

\section{The design of antenna array and related works}

For more than a century, especially after the Second World War, antenna theory, design, and application have been rapidly developed. Antenna array is an important type of antenna [30]. An antenna system consisting of two or more discrete antennas is called an antenna array. There are many kinds of antenna arrays, according to the arrangement of elements, wired array, and plane array $[31,32]$.

A linear array is an antenna array consisting of a plurality of units separated from each other and centered on a line [33]. A linear array is divided into uniform linear array and non-uniform linear array: uniform linear array means the equal distance between the adjacent antenna unit and the constant incentive phase difference between adjacent units, that is to say each unit is a linear array excited by the law of equality. Non-uniform linear array refers to that the distance between the adjacent antenna elements is not equal, and each element is a linear array inspired by the law of non-synchronous progressive phase.

Let us consider the design of the linear antenna array. The array consists of many elements aligning to a line shape. Previously, array elements were assumed to be distributed with equal distance. This arrangement simplifies the geometric structure and saves computation. On the other hand, recent researches show that unequal distance distribution is apt to provide better QoS. Thus, an unequal distance distribution structure is considered in the paper. The design model is:

$$
\begin{array}{ll}
\max & f=\left|\frac{E(u)}{P M}\right| \\
\text { s.t. } & E(u)=\sum_{i=1}^{G} I_{i} e^{j \varphi_{i}} e^{j k u x_{i}} d_{i} \\
& u=\cos \theta-\cos \theta_{0} \\
& d_{i}-d_{k} \geq d_{c}, 1 \leq k<i \leq G, \\
& d_{c}>0
\end{array}
$$

where $k=2 \pi / \lambda, \lambda$ is the wavelength, $G$ is the number of array elements, and PM is the peak value of the main lobe of antenna array. $\theta_{0}$ is the incoming direction of the main beam. $d_{c}$ is the minimum distance between two successive elements. $I_{i}$ and $\varphi_{i}$ are the amplitude of excitation and phase, respectively.

In model (1), $d_{i}$ could be any real numbers such that $d_{i}-d_{i-1} \geq d_{c}$ and $d_{i+1}-d_{i} \geq d_{c}$. Moreover, $I_{i}$ and $\varphi_{i}$ are also variables to be defined by users. These can be set as parameters in the antenna array system.

\section{The CAPSO method}

The CAPSO method is composed by four stages. They are initialization, updating particles' velocity and position, solution evaluation, and updating parameters as shown in Fig. 1. Initially, CAPSO begins with a swarm of particles, where the number of particles is denoted as $N P$. In general, each particle is a solution for model (1). Hence, the CAPSO algorithm is initialized with $N P$ feasible solutions, which can be easily implemented in parallel [34].

When updating particles' velocity and position stage, $\mathbf{v}_{i}(1 \leq i \leq N P)$ is updated by the following formula:

$$
v_{i j}(t+1)=\varphi\left(v_{i j}(t)\right)+c_{1} r_{1} d_{i j}^{p}(t)+c_{2} r_{2} d_{i j}^{g}(t),
$$

where index $i$ refers to the $i$ th solution and $j$ refers to the $j$ th variable of the $i$ th solution, $t$ is the iteration number. Parameter $\varphi$ is called contraction factor controlling the velocities of particles. Parameters $c_{1}$ and $c_{2}$ are called accelerating factor, which have to be set by users. Numbers $r_{1}$ and $r_{2}$ are two random numbers between 0 and 1 , which are generated at each iteration. $d_{i j}^{p}$ is:

$$
d_{i j}^{p}(t)=p_{i j}(t)-x_{i j}(t)
$$

where $p_{i j}$ refers to the best personal solution found by particle $i . d_{i j}^{g}$ is:

$$
d_{i j}^{g}(t)=g_{j}(t)-x_{i j}(t),
$$




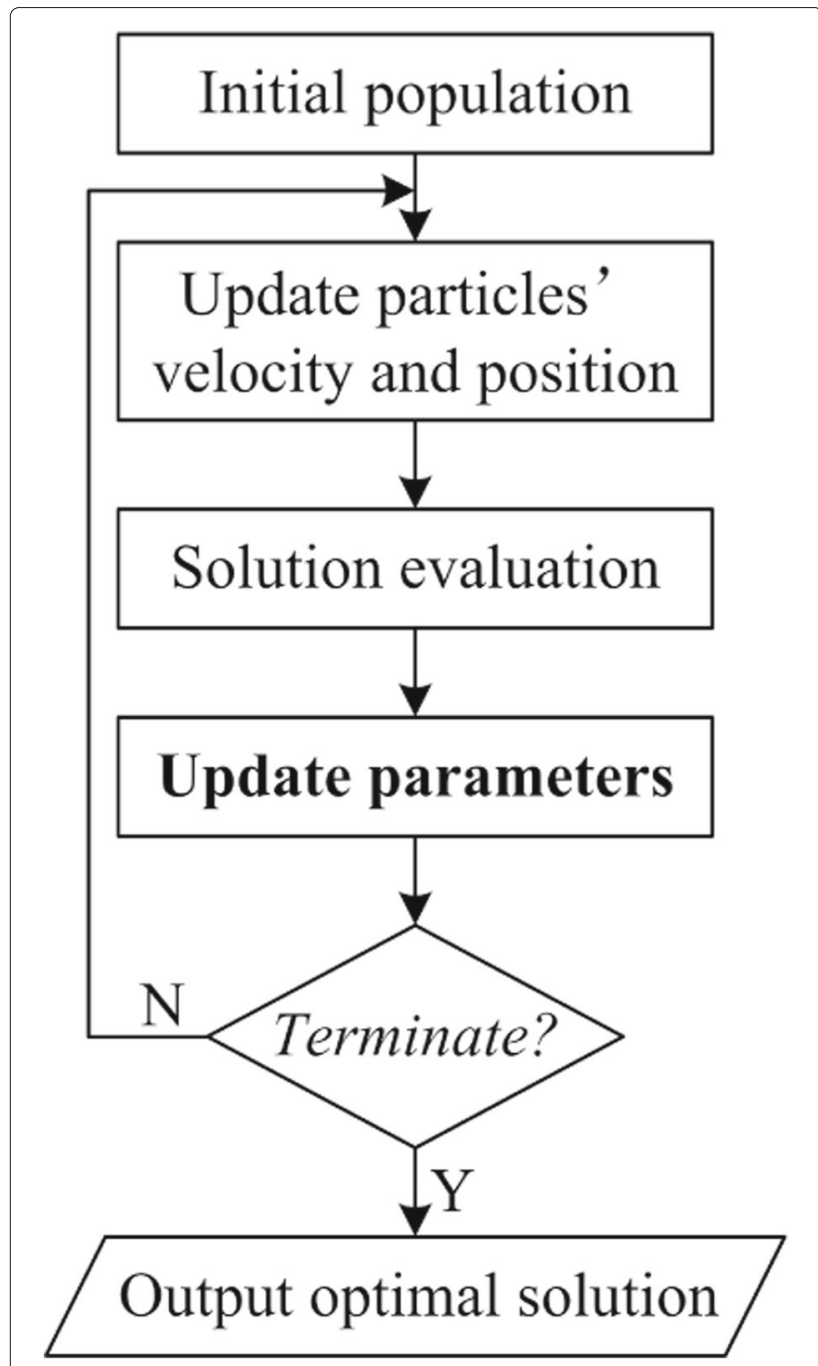

Fig. 1 Flow chart of the CAPSO method

where $g_{j}$ refers to the best solution found by all particles. Finally, new solution is produced by:

$$
x_{i j}(t+1)=x_{i j}(t)+v_{i j}(t+1),
$$

It can be seen from (2), $\varphi, c_{1}$ and $c_{2}$ are algorithmic parameters as well as NP.

$\varphi$ is called contraction factor controlling the velocities of particles. $c_{1}$ and $c_{2}$ control the extent that particle $i$ flying toward local best solution or global best solution. These three parameters are adapted as follows:

$$
c_{1}=c_{1}^{\max }+\frac{\left(c_{1}^{\max }-c_{1}^{\min }\right) \times t}{t^{\max }},
$$

where $c_{1}^{\max }$ and $c_{1}^{\min }$ are respectively the maximal and minimal vales for $c_{1} \cdot t^{\max }$ is the maximal number of iterations. Similarly, $c_{2}$ is adapted:

$$
c_{2}=c_{2}^{\max }+\frac{\left(c_{2}^{\max }-c_{2}^{\min }\right) \times t}{t^{\max }},
$$

$\varphi$ is adapted:

$$
\varphi=\frac{2}{\left|2-\left(c_{1}+c_{2}\right)-\sqrt{\left(c_{1}+c_{2}\right)^{2}-4\left(c_{1}+c_{2}\right)}\right|},
$$

The adaptive formula of $\varphi$ is previously studied in [35].

In the CAPSO method, parameters $\varphi, c_{1}$ and $c_{2}$ are all adapted based on (6), (7), and (8). $c_{1}$ and $c_{2}$ are based on the iteration process of the method. Their values gradually decrease from $c_{i}^{\max }$ to $c_{i}^{\min }(i=1,2)$. Based on (8), $\varphi$ also decreases along with iteration. Note that $c_{1}+c_{2} \geq 4$ is required to fulfill the root operation. Thus, the CAPSO method performs a large search step size in the former iteration process, while performing small search step size in the later iteration process. The procedures of CAPSO are identical to the philosophy of heuristic methods [36, 37].

\section{Numerical experiment settings}

The CAPSO method will be studied on toy functions and then applied to solve antenna array designs in this section.

The following toy functions are used:

(1) $f_{1}(\mathbf{x})=-100\left(x_{1}^{2}-x_{2}^{2}\right)-\left(1-x_{1}^{2}\right)$;

(2) $f_{2}(\mathbf{x})=-\left(4 x_{1}^{2}-2.1 x_{1}^{4}+x_{1}^{6} / 3+x_{1} x_{2}-4 x_{2}^{2}+x_{2}^{4}\right)$;

Both functions are unconstrained maximization problems with two independent variables, which can take any real numbers. The CAPSO method is independently tested 25 times on each function with $t^{\max }=10,000$.

In the simulation of antenna array designs, excitation amplitude $I_{i}$ and phase $\varphi_{i}$ for element $i$ are set to constant. $d_{i}$ between adjacent elements are independent variables and computed based on distance of adjacent elements. The length of array $L$ is set to $N \lambda$. Hence, with different numbers of elements $G, L$ is automatically changed. The design problem is then scalable and can be tested with any number of array elements.

\section{Results and discussion}

Let us first study the convergence rate of the CAPSO method. Standard PSO [35], IPSO [37], and APSO [35] are used for comparison. In the simulation, $N P$ is set to 40 following [35]. The results are presented in Table 1. This table gives the average number of iterations that a method finds global optimal solution with threshold $10^{-8}$. In terms of the mean number of iterations, the CAPSO method converges to global optimum faster than the other methods. In terms of standard deviation (std), the CAPSO method has larger std than standard PSO on both functions. The std of CAPSO is also larger than that of IPSO and APSO for $f_{1}$, but the std of CAPSO is much smaller than that of IPSO and APSO for $f_{2}$. The last column of Table 1 gives the hypothesis test of CAPSO versus PSO, IPSO, and APSO. The $p$ values less than 0.05 means a significant difference between two methods. It can be seen 
Table 1 Result comparison of the CAPSO method versus standard PSO, IPSO, and APSO methods

\begin{tabular}{lllll}
\hline Method & $f(\cdot)$ & Mean & std & $p$ \\
\hline PSO & $f_{1}$ & 1099.20 & 434.68 & $2.16 \mathrm{E}-7$ \\
& $f_{2}$ & 4033.60 & 32.00 & $7.31 \mathrm{E}-6$ \\
IPSO & $f_{1}$ & 908.80 & 222.27 & $2.37 \mathrm{E}-7$ \\
& $f_{2}$ & 3072.00 & 448.85 & $1.15 \mathrm{E}-7$ \\
APSO & $f_{1}$ & 939.20 & 210.12 & $2.36 \mathrm{E}-7$ \\
& $f_{2}$ & 3172.80 & 446.58 & $1.20 \mathrm{E}-7$ \\
CAPSO & $f_{1}$ & 449.60 & 553.93 & N/A \\
& $f_{2}$ & 2004.80 & 46.64 & N/A \\
\hline
\end{tabular}

Hypothesis test decides significant difference of results of two methods and is shown in the $p$ column. N/A means the data is not available

that CAPSO is significantly better than the other three methods.

Parameter NP is not very sensitive to problem types. For PSO and its improved methods, $N P$ is often set to 40 , though some researches used 30 or 50 for large-scale problems. In this paper, six NP values ranging from 10 to 60 are tested. The results on $f_{1}$ and $f_{2}$ are presented in Fig. 2. The function values are normalized to clearly present the results. It can be seen from the figure that $N P=30$ or $N P=40$ are much better than other values. Moreover, $N P=30$ gives the best results on both functions. Hence, the default setting of CAPSO is $N P=30$, which will be used to solve the antenna array design.

In the simulation of antenna array designs, $t^{\max }=$ $1000 G$ and the CAPSO method is executed 25 times to gain an average performance. Figures 3, 4, and 5 respectively show the best execution of 25 times for $G=10$, $G=30$, and $G=50$.

Figure 3 shows a far field pattern associated with the optimal solution when $G=10$. It can be seen from the figure that the main lobe is about $10 \mathrm{~dB}$ better than the nearest side lobe. Moreover, the range of the main lobe is about $5^{\circ}$ around $\theta_{0}=90^{\circ}$. Thus, the attained antenna array $G=10$ is a wide-band case.

Figure 4 shows a far field pattern associated with the optimal solution when $G=30$. It can be seen from the figure that the main lobe is about $14 \mathrm{~dB}$ better than the nearest side lobe. Moreover, the range of main lobe is about $2^{\circ}$ around $\theta_{0}=90^{\circ}$. Thus, the attained array $G=30$ is narrower than the array $G=10$.

Figure 5 shows a far field pattern associated with the optimal solution when $G=50$. It can be seen from the figure that the main lobe is about $16.5 \mathrm{~dB}$ better than the nearest side lobe. Moreover, the range of main lobe is about $2^{\circ}$ around $\theta_{0}=90^{\circ}$. Thus, the attained array $G=50$

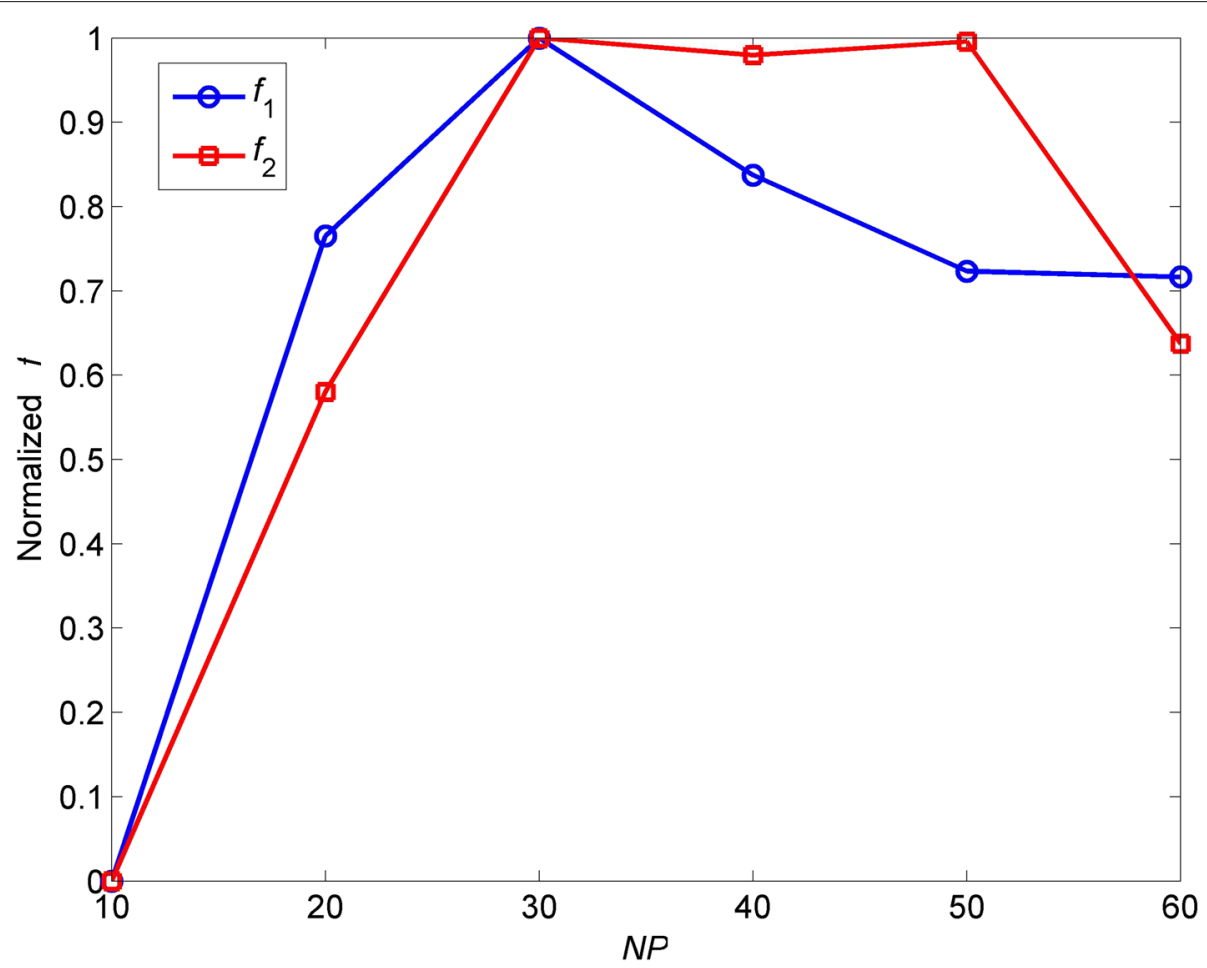

Fig. 2 Performance of the CAPSO method with $N P=10,20,30,40,50$, and 60 for $f_{1}$ and $f_{2}$ 


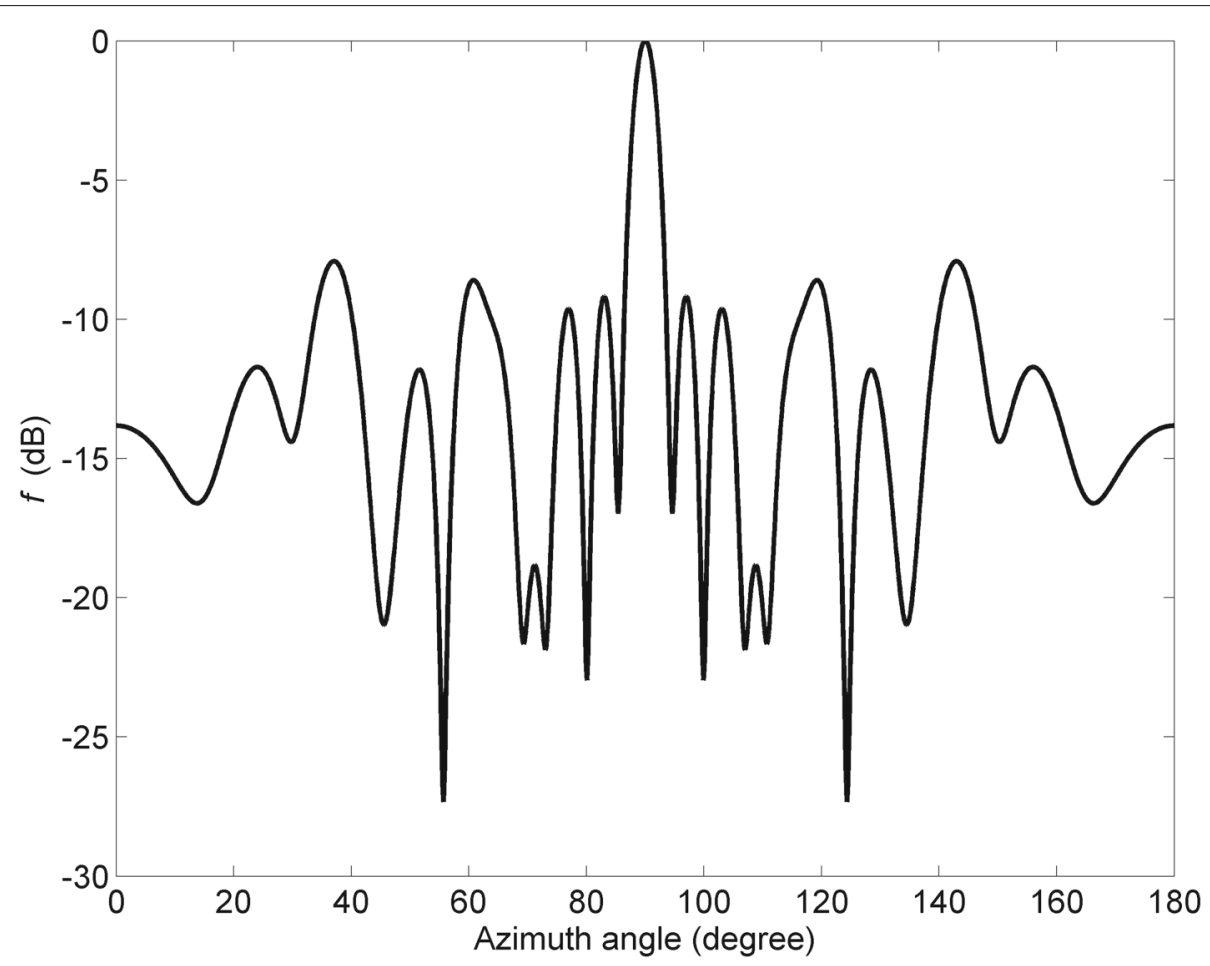

Fig. 3 Optimal result obtained by the CAPSO method for $G=10$

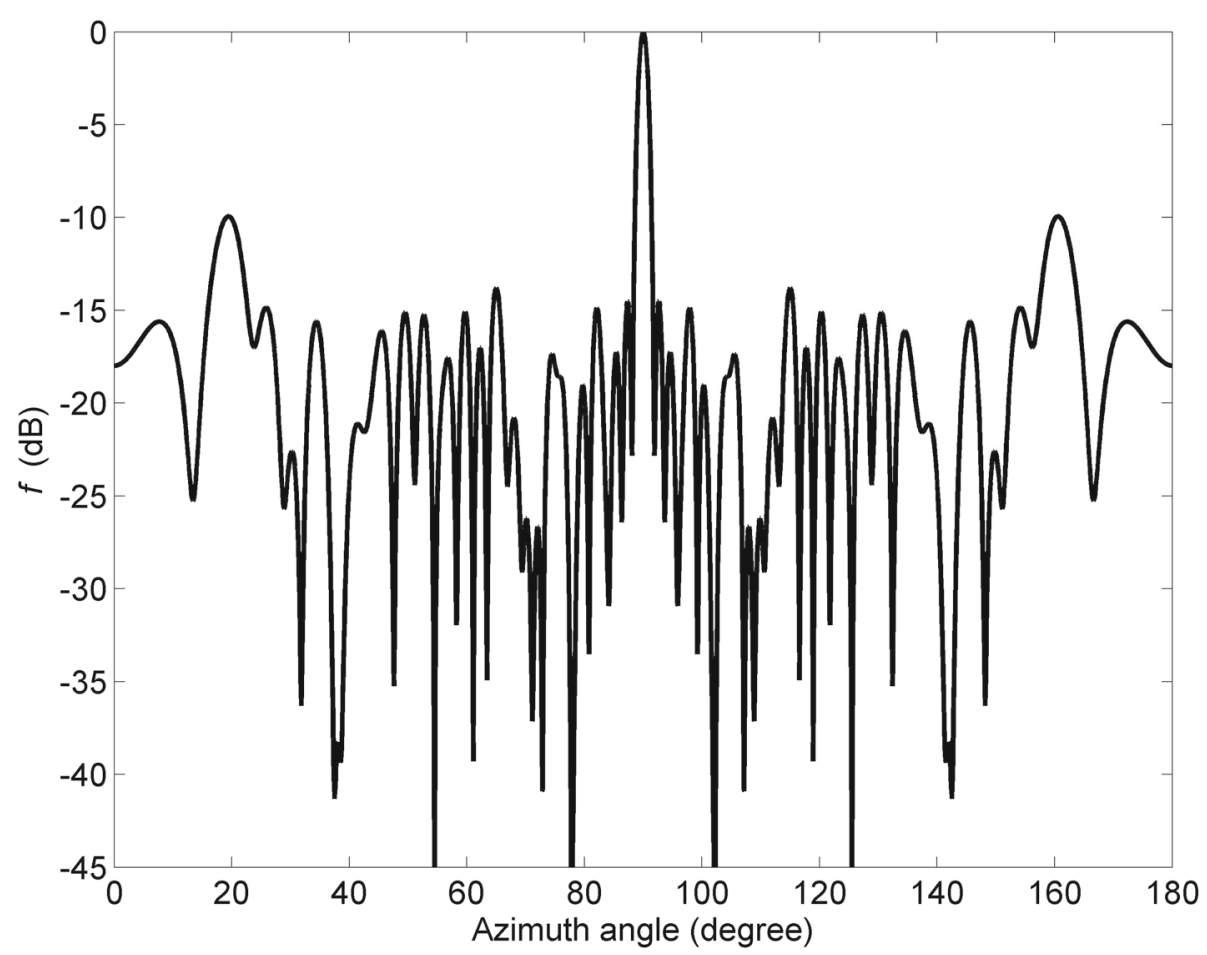

Fig. 4 Optimal result obtained by the CAPSO method for $G=30$ 


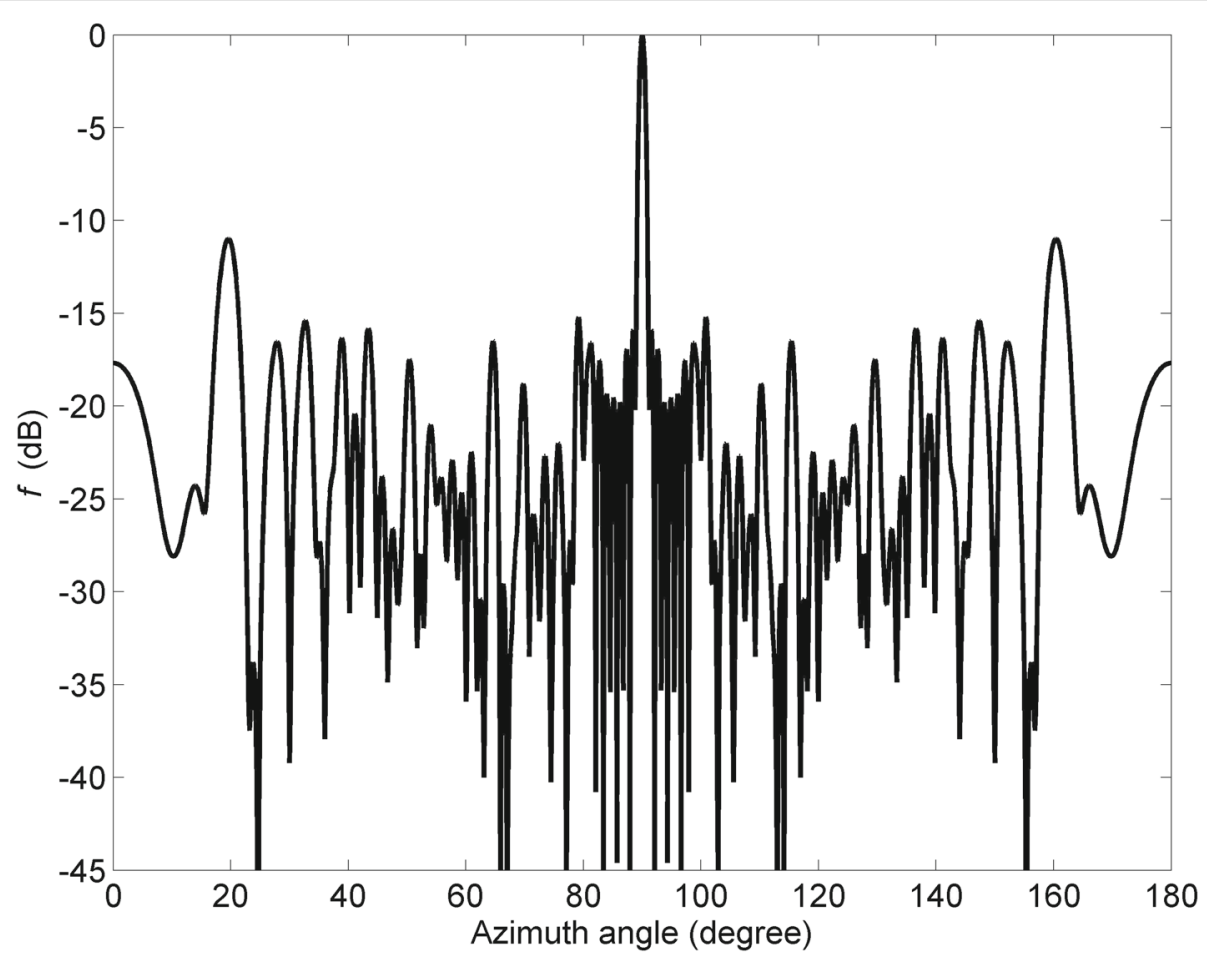

Fig. 5 Optimal result obtained by the CAPSO method for $G=50$

is similar as the array $G=30$, but is narrower than the array $G=10$.

From the above simulation, it can be seen that the gain of antenna array increases with the number of elements. However, the band width decreases with the number of elements from 10 to 30 array elements. The band width does not decrease with array elements from 30 to 50 . Because the tested antenna array problem scales from 10 to 50 array elements, the results show that our method is suitable to such designs.

\section{Conclusion}

Radio waves are widely used in communication, broadcasting, target detection, navigation, and other fields. The transmission and reception of radio waves depend on antennas.

This paper focuses on how to optimize the positions of antenna array elements so as to maximize the performance of antenna in transmitting and receiving data. The far field pattern and side lobe level value are main metrics of the performance of antenna array. The proposed CAPSO method is based on contraction factor adaptation. Accelerating factors are also adapted.

A numerical experiment is tested on two toy functions for analyzing the convergence rate of the CAPSO method. Compared with three other methods, CAPSO can achieve good convergence performance. The simulation on antenna array shows that the CAPSO method is able to find good solution, though when the number of elements becomes large, the band width decreases. The band width does not decrease too much from 30 elements to 50 elements. Because the tested antenna array problem is scalable, the results show that our method is suitable to such designs.

\section{Abbreviations}

5G: Fifth generation; CAPSO: Contraction adaptive particle swarm optimization; GA: Genetic algorithm; IPSO: Improved particle swarm optimization; MIMO: Multiple input multiple output; NP: Number of particles; PSO: Particle swarm optimization; QoS: Quality of service

\section{Acknowledgements}

Not applicable.

\section{Funding}

This research was supported in part by the National Natural Science Foundation of China (Project No. 61601329, 61603275, 61601328), the Tianjin Higher Education Creative Team Funds Program, and the Applied Basic Research Program of Tianjin (Project No. 18JCYBJC16100).

\section{Availability of data and materials}

Data sharing not applicable to this article as no datasets were generated or analysed during the current study.

\section{Authors' contributions}

XZ proposes the algorithm framework based on PSO and writes most of this paper except that XZ writes the antenna array model section. DL is in charge of the computer simulation of the algorithm and collection of the results. YW organizes the whole paper as well as contributes useful discussion. All authors read and approved the final manuscript.

\section{Authors' information}

Not applicable. 


\section{Competing interests}

The authors declare that they have no competing interests.

\section{Publisher's Note}

Springer Nature remains neutral with regard to jurisdictional claims in published maps and institutional affiliations.

Received: 5 December 2018 Accepted: 21 February 2019

Published online: 11 March 2019

\section{References}

1. F. Zhao, W. Wang, H. Chen, Q. Zhang, Interference alignment and game-theoretic power allocation in MIMO heterogeneous sensor networks communications. Signal Process. 126, 173-179 (2016)

2. K. Yang, L. Wang, S. Wang, X. Zhang, Optimization of resource allocation and user association for energy efficiency in future wireless networks. IEEE Access. 5, 16469-16477 (2017)

3. Q. Liang, Situation understanding based on heterogeneous sensor networks and human-inspired favor weak fuzzy logic system. IEEE Syst. J. 5(2), 156-163 (2011)

4. Q. Liang, X. Cheng, S. Huang, D. Chen, Opportunistic sensing in wireless sensor networks: theory and applications. IEEE Trans. Comput. 63(8), 2002-2010 (2014)

5. F. Zhao, X. Sun, H. Chen, R. Bie, Outage performance of relay-assisted primary and secondary transmissions in cognitive relay networks. EURASIP J. Wirel. Commun. Netw. 2014, 60 (2014)

6. J.WU, C. ZENG, J. SUN, Research and application of wireless intelligent network monitoring smog system based on stm32f407. J. Tianjin Normal Univ. (Nat. Sci. Ed.) 37(6), 62-66 (2017)

7. X. Wang, Q. Liang, On the throughput capacity and performance analysis of hybrid wireless networks over fading channels. IEEE Trans. Wirel. Commun. 12(6), 2930-2940 (2013)

8. L. Han, J. Mu, Y. Wang, J. Gao, Performance analysis of multi-hop full-duplex decode-and-forward relaying. Ad Hoc Netw. 58, 54-61 (2017)

9. F. Zhao, L. Wei, H. Chen, Optimal time allocation for wireless information and power transfer in wireless powered communication systems. IEEE Trans. Veh. Technol. 65(3), 1830-1835 (2016)

10. N. W. Liu, L. Zhu, X. Zhang, W. W. Choi, A wideband differential-fed dual-polarized microstrip antenna under radiation of dual improved odd-order resonant modes. IEEE Access. 5, 23672-23680 (2017)

11. G. Ma, B. Huang, Optimization of process parameters of stamping forming of the automotive lower floor board. J. Appl. Math. 2014, 470320 (2014)

12. J. Zhu, B. Hao, A new class of smoothing functions and a smoothing newton method for complementarity problems. Optimization Lett. 7(3), 481-497 (2013)

13. J. Tang, G. He, L. Fang, A new non-interior continuation method for second-order cone programming. J. Numer. Math. 21(4), 301-323 (2013)

14. R. Guo, Z. Zhang, X. Liu, C. Lin, Existence, uniqueness, and exponential stability analysis for complex-valued memristor-based bam neural networks with time delays. Appl. Math. Comput. 311, 100-117 (2017)

15. Q. Jia, W. K. S. Tang, Consensus of multi-agents with event-based nonlinear coupling over time-varying digraphs. IEEE Trans. Circ. Syst.-II: Express Briefs. to appear (2018). https://doi.org/10.1109/TCSII.2018.2790582

16. Y. S. C. Liu, PingHan: A new infeasible-interior-point algorithm for linear programming over symmetric cones. Acta Math. Applicatae Sin. Engl. Ser. 33(3), 771-788 (2017)

17. J. Zhu, B. Hao, A new noninterior continuation method for solving a system of equalities and inequalities. J. Appl. Math. 2014, 592540 (2014)

18. Z. Z. Feng, L. Fang, G. He, An iteration primal-dual path-following method based on wide neighbourhood and large update, for second-order cone programming. Optimization. 63(5), 679-691 (2014)

19. J. Tang, G. He, L. Dong, L. Fang, J. Zhou, A smoothing newton method for the second-order cone complementarity problem. Appl. Math. 58(2), 223-247 (2013)

20. J. Yu, M. Li, Y. Wang, G. He, A decomposition method for large-scale box constrained optimization. Appl. Math. Comput. 231(12), 9-15 (2014)

21. D. Bin-Chao, T. Chen, B. Xin, Parallel and cyclic algorithms for quasi-nonexpansives in hilbert space. Abstr. Appl. Anal. 2012, 218341 (2012)

22. Y. Lou, S. Y. Yuen, Non-revisiting genetic algorithm with adaptive mutation using constant memory. Memetic Comput. 8(3), 189-210 (2016). https://doi.org/10.1007/s12293-015-0178-6
23. S. Pang, T. Li, F. Dai, M. Yu, Particle swarm optimization algorithm for multisalesman problem with time and capacity constraints. Appl. Math Inf. Sci. 7(6), 2439-2444 (2013)

24. X. Zhang, X. Zhang, Improving differential evolution by differential vector archive and hybrid repair method for global optimization. Soft Comput. 21(23), 7107-7116(2017)

25. B. Zhu, X. Xia, Z. Wu, Evolutionary game theoretic demand-side management and control for a class of networked smart grid. Automatica. 70, 94-100 (2016)

26. Z. Wu, T. W. S. Chow, Neighborhood field for cooperative optimization. Soft Comput. 17(17), 819-834 (2013)

27. F. Zhao, H. Nie, H. Chen, Group buying spectrum auction algorithm for fractional frequency reuses cognitive cellular systems. Ad Hoc Netw. 58 239-246 (2017)

28. Y. Zou, G. He, On the uniqueness of solutions for a class of fractional differential equations. Appl. Math. Lett. 74, 68-73 (2017)

29. X. Jin, Y. Liang, D. Tian, F. Zhuang, Particle swarm optimization using dimension selection methods. Appl. Math. Comput. 219(10), 5185-5197 (2013)

30. F. Zhao, B. Li, H. Chen, X. LV, Joint beamforming and power allocation for cognitive MIMO systems under imperfect CSI based on game theory. Wirel. Pers. Commun. 73(3), 679-694 (2013)

31. X. Chen, P. Y. Qin, Y. J. Guo, G. Fu, Low-profile and wide-beamwidth dual-polarized distributed microstrip antenna. IEEE Access. 5, 2272-2280 (2017)

32. X. Zhang, X. Zhang, Circular antenna design by adaptive position inheritance artificial bee colony algorithm. Phys. Commun. 25, 369-375 (2017)

33. X. Zhang, X. Zhang, L. Wang, Antenna design by an adaptive variable differential artificial bee colony algorithm. IEEE Trans. Magn. 54(3) $7201704(2018)$

34. C. Y. Han, F. Y. Zheng, T. D. Guo, G. P. He, Parallel algorithms for large-scale linearly constrained minimization problem. Acta Math. Applicatae Sin Engl. Ser. 30(3), 707-720 (2014)

35. J. Kennedy, R. C. Eberhart, Y. Shi, Swarm Intelligence. (Morgan Kaufman, San Francisco, U.S., 2001)

36. X. Zhang, S. Y. Yuen, A directional mutation operator for differential evolution algorithms. Appl. Soft Comput. 30, 529-548 (2015)

37. Z. S. Zhao, X. Feng, Y. Y. Lin, F. Wei, S. K. Wang, T. L. Xiao, M. Y. Cao, Z. G. Hou, M. Tan, Improved rao-blackwellized particle filter by particle swarm optimization. J. Appl. Math. 2013, 302170 (2013)

\section{Submit your manuscript to a SpringerOpen ${ }^{\circ}$ journal and benefit from:}

Convenient online submission

- Rigorous peer review

- Open access: articles freely available online

- High visibility within the field

Retaining the copyright to your article

Submit your next manuscript at springeropen.com 